\title{
Research Paper \\ Leisure Spending Patterns and their Relationship with Mental Health in the Elderly in Iran
}

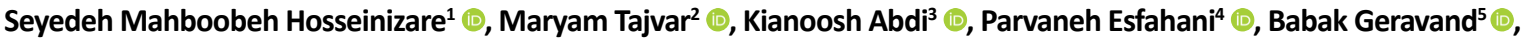 \\ *Abolghasem Pourreza ${ }^{6}$ (1)
}

1. Social Determinants of Health Research Center, University of Social Welfare and Rehabilitation Sciences, Tehran, Iran.

2. Department of Health Management and Economics, School of Public Health, Tehran University of Medical Sciences, Tehran, Iran.

3. Department of Rehabilitation Management, University of Social Welfare and Rehabilitation Sciences, Tehran, Iran.

4. Department of Health care Services Management, School of Public Health, Zabol University of Medical Sciences, Zabol, Iran.

5. Department of Health Care Management, Faculty of Management and Accounting, Islamic Azad University, South Tehran Branch, Tehran, Iran.

6. Department of Health Education and Health Promotion, School of Public Health, Tehran University of Medical Sciences, Tehran, Iran.

\begin{tabular}{|c|c|}
\hline $\begin{array}{l}\text { Use your device to scan } \\
\text { and read the article online }\end{array}$ & Chtation: Hosseinizare SM, Tajvar M, Abdi K, Esfahani P, Geravand B, Pourreza A. [Leisure Spending Patterns and their \\
\hline 口: & $\begin{array}{l}\text { Relationship with Mental Health in the Elderly in Iran (Persian)]. Iranian Journal of Ageing. 2020; 15(3):366-379. https://doi. } \\
\text { org/10.32598/sija.15.3.2704.1 }\end{array}$ \\
\hline arterp & dol'https://doi.org/10.32598/sija.15.3.2704.1 \\
\hline
\end{tabular}

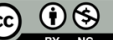

Received: 07 May 2019

Accepted: 13 Jan 2020

Available Online: 01 Oct 2020

Key words:

Mental health, Lei-

sure activities, Aged

\section{A B S T R A C T}

Objectives Older adults need vitality to survive. Entertainment and activity for the elderly creates a lively life, boost their self-esteem, and saves them from being inactive and unproductive, which will ultimately improve their mental health. This study aims was to determine the relationship between leisure spending patterns and mental health in the elderly

Methods \& Materials This descriptive-analytical study was carried out on 413 elderly people aged $\geq 60$ years, who were selected using a cluster random sampling method from four districts of Tehran, Iran. Data were collected using the standard General Health Questionnaire, and a researcher-made Leisure Spending Scale. The collected data were analyzed in SPSS V. 21 software using descriptive statistics (frequency, standard deviation) and statistical tests including Spearman correlation test and regression analysis.

Results The most frequent leisure activity was TV watching. There was a significant relationship between recreational and religious activities of elderly and their mental health $(P<0.0001)$. Among the confounding factors, physical health had the most impact.

Conclusion Given the significant relationship of recreational and religious activities with the mental health of the elderly, planners should take the role of mosques, hosayniyas, and leisure facility providers such as municipalities, seriously. On the other hand, considering the impact of physical health on the relationship of leisure spending with mental health, it is necessary to provide opportunities to the elderly to participate in sports and recreational activities.

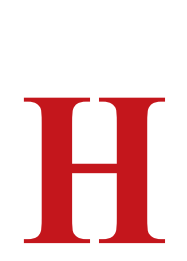

\section{Extended Abstract}

\section{Introduction}

ealthy aging is the right of all human beings, which highlights the need for preventing its related problems. The elderly have more leisure time after retirement. Leisure time gives them the opportunity to gain strength, confidence, creativity, and physical and mental health [1]. The elderly face many risks such as retirement/unemployment, reduced income, loneliness, lack of social support, industrialization of communities, loss of spouse, separation from children, emotional/psychological separation of family members,

\section{* Corresponding Author:}

Abolghasem Pourreza, PhD.

Address: Department of Health Education and Health Promotion, School of Public Health, Tehran University of Medical Sciences, Tehran, Iran Tel: +98 (912) 6215044

E-mail: abolghasemp@yahoo.com 
and ultimately reduced physical and mental function. Creating opportunities for the elderly to have social participation, including healthy leisure time, can be an effective step in addressing these risks and threats and improving their quality of life. The purpose of this study is to investigate the effect of leisure-spending patterns on the mental health of the elderly.

\section{Methods \& Materials}

This is a descriptive-analytical study with cross-sectional design conducted in 2018. The participants were 430 community-dwelling elderly aged $\geq 60$ years in Tehran, Iran. Inclusion criteria were: No dementia and any critical physical condition, ability to understand and respond to questions, and willingness to participate in the study. Multistage random cluster sampling method was used for sampling. Data collection tools were a demographic form, a researchermade Leisure Spending Questionnaire, the 36-Item Short Form Survey (SF-36) to assess the physical health of the elderly [3], and the 15-item General Health Questionnaire (GHQ-15) to assess their mental health [2].

Descriptive statistics and analytical tests were conducted in SPSS V. 21 software. In this study, the dependent vari- able was mental health and the main independent variable was leisure spending. The descriptive statistics describe the frequency and standard deviation of demographic factors, leisure spending patterns, and mental health of the elderly. Kolmogorov-Smirnov test was used to measure the normality of leisure spending and mental health scores. Based on its Results, Spearman correlation test was used to investigate the correlation between different patterns of leisure spending and the mental health of the elderly. Multiple regression analysis was also used to examine the simultaneous effect of leisure spending patterns on the mental health. Since the Results of Saberian et al. and Ilali et al. showed the significant relationship of age, gender, education, income and physical health with leisure spending and mental health $[4,5]$, in this study, the effects of age, gender, education, financial status, and physical health on the regression model as confounding factors were controlled.

\section{Results}

In this study, out of 430 participants, the data from 413 were used for statistical analysis and 17 were excluded. Most of them ( $\mathrm{n}=408,98.8 \%$ ) were reported to watch television during their leisure time. In terms of gender, Results showed that the participation of women in recreational,

Table 1. Multiple regression coefficients (Dependent variable: Mental health)

\begin{tabular}{|c|c|c|c|c|c|c|c|c|c|c|c|c|}
\hline \multirow{2}{*}{$\begin{array}{l}\text { Leisure Spend- } \\
\text { ing Patterns }\end{array}$} & \multicolumn{3}{|c|}{ Model 1} & \multicolumn{3}{|c|}{ Model 2} & \multicolumn{3}{|c|}{ Model 3} & \multicolumn{3}{|c|}{ Model 4} \\
\hline & Beta & $\mathbf{P}$ & $\mathbf{F}$ & Beta & $\mathbf{P}$ & $\mathbf{F}$ & Beta & $\mathbf{P}$ & $\mathbf{F}$ & Beta & $\mathbf{P}$ & $\mathbf{F}$ \\
\hline Recreational & -0.151 & 0.026 & & -0.019 & 0.760 & & - & - & & - & - & \\
\hline Sports & -0.075 & 0.231 & & 0.002 & 0.977 & & - & - & & - & - & \\
\hline Social & -0.022 & 0.696 & & -0.028 & 0.588 & & - & - & & - & - & \\
\hline Arts & -0.097 & 0.087 & 532.7 & -0.092 & 0.070 & 13.17 & & & & & - & \\
\hline Cultural & -0.052 & 0.313 & & -0.032 & 0.536 & & - & - & & - & - & \\
\hline Religeous & -0.117 & 0.015 & & -0.097 & 0.030 & & - & - & & - & - & \\
\hline Overall score & - & - & & - & - & & -0.351 & -0.000 & 57.88 & -0.173 & 0.000 & 26.24 \\
\hline Gender & - & - & & -0.060 & 0.233 & & - & - & & -0.055 & 0.258 & \\
\hline Age & - & - & & -0.034 & 0.490 & & - & - & & -0.027 & 0.569 & \\
\hline Marital status & - & - & & 0.099 & 0.053 & & - & - & & 0.089 & 0.068 & \\
\hline Education & - & - & & 0.015 & 0.777 & & - & - & & 0.016 & 0.739 & \\
\hline Financial status & - & - & & 0.108 & 0.016 & & - & - & & 0.098 & 0.027 & \\
\hline Physical health & - & - & & -0.418 & 0.000 & & - & - & & -0.414 & 0.000 & \\
\hline
\end{tabular}


sports, cultural, and social media activities was significantly lower compared to men. However, women were more involved in social and religious activities. In terms of age, the Results showed that the participation of the elderly in recreational, social and arts activities was significantly reduced with the increase of age.

According to Spearman correlation test Results, all patterns of leisure spending were significantly correlated with the mental health, except the resting and social media activities. To examine the simultaneous effect of leisure spending patterns on the mental health, multiple regression analysis was used after excluding resting and social media activities from the model. The Results are presented in Table 1, considering that the higher GHQ scores indicate the lower mental health. According to the regression model 2 , the recreational activity lost its significant effect in the presence of confounding factors. Among the confounding variables, physical health had the most negative effect $(\beta=$ -0.418). In the regression model 3, leisure spending was inversely correlated with the GHQ score in the absence of confounding factors. In the regression model 4, leisure spending was inversely correlated with GHQ in the presence of confounding factors $(\beta=-0.173$ ), but its effect was lower than in the absence of confounding factors. Most of effect in the confounding factors was related to physical health; however, financial status, education, and age were also important factors.

\section{Conclusion}

The Results of this study showed that physical health is an effective confounding factor in the relationship of leisure spending with the mental health of the elderly. Since physical activity plays an important role in enhancing the ability and prevention of diseases, it is important to address the leisure issues that involve physical activity. Policymakers' awareness of the current situation, identifying the important needs of the elderly, considering the welfare facilities, and encouraging the elderly to participate in sports activities are effective steps to improve their physical health and thus participate in more leisure programs, which help improve their mental health. According to the Results, recreational and religious activities had a significant relationship with the mental health of the elderly. Therefore, more detailed plans for mosques and volunteered activities are suggested for improving both the mental and physical health of the elderly. Providing facilities in these places that can engage the elderly in recreational activities can improve their mental health. Furthermore, with appropriate planning in the neighborhood level by the Municipality, necessary actions can be taken.

\section{Ethical Considerations}

\section{Compliance with ethical guidelines}

This study was approved by the Ethics Committee of Tehran University of Medical Sciences (Code: IR.TUMS.VCR.REC.1395.320).

\section{Funding}

This research did not receive any specific grant from funding agencies in the public, commercial, or not-for-profit sectors.

\section{Authors' contributions}

All authors contributed equally in preparing all parts of the research.

\section{Conflicts of interest}

The authors declared no conflict of interest. 


\title{
كذران اوقات فراغت و ارتباط آن با سلامت روان سالمندان شهر تهران در سال ITqV
}

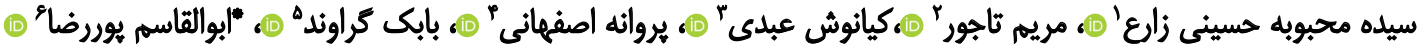

1. امركز تحقيقات عوامل اجتماعى مؤثر بر سلامت، دانشكاه علوم بهزيستى و توانبخشى، تهران، ايران.

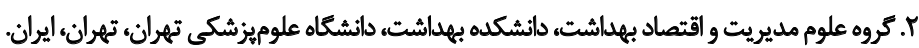

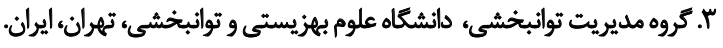

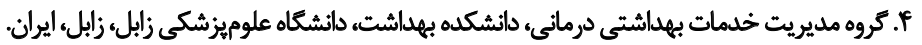

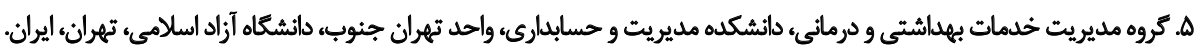

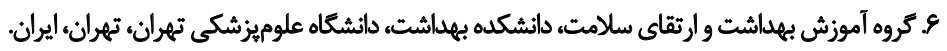

\section{حكיد}

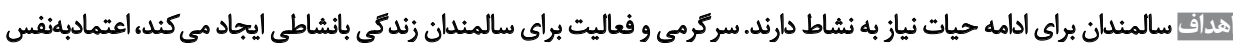

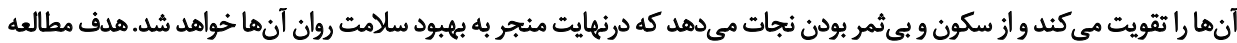

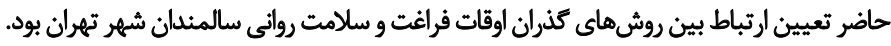

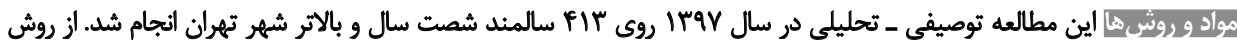

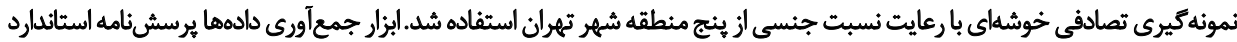

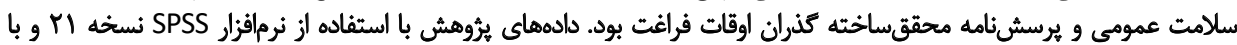

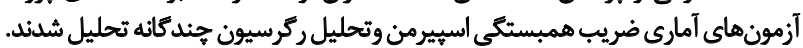

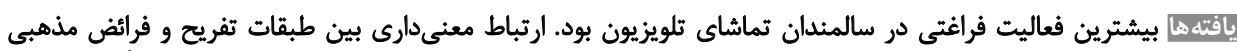

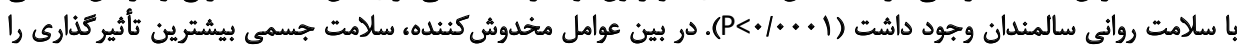
داشت.

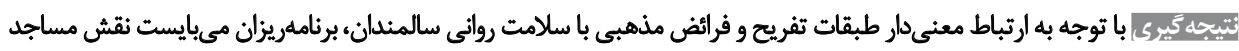

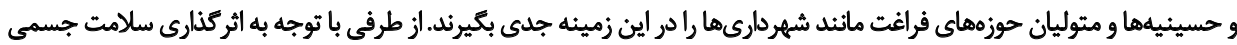

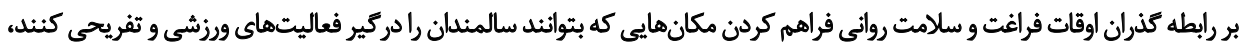

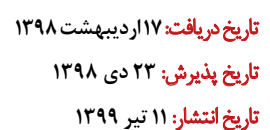

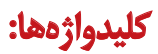

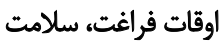

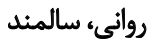

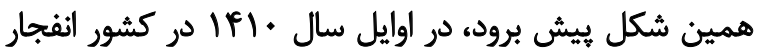

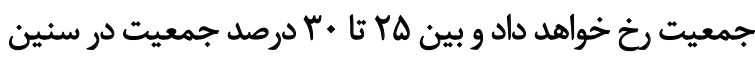

مقدمه

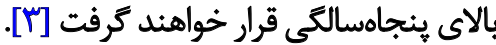

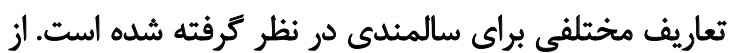

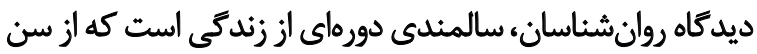

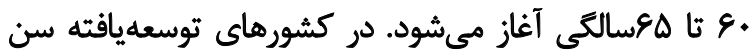

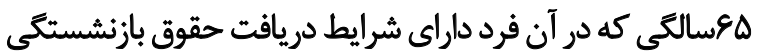

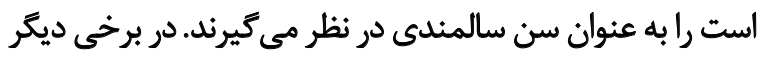

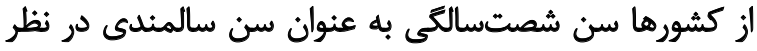

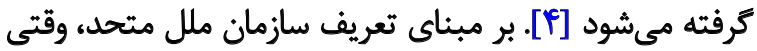

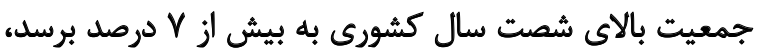

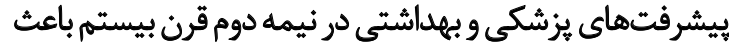

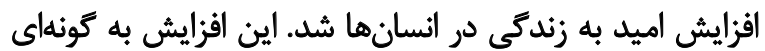

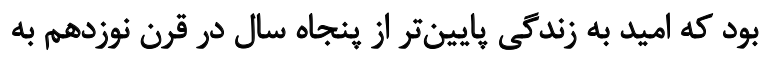

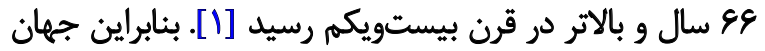

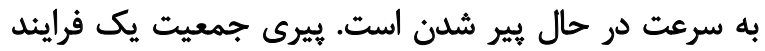

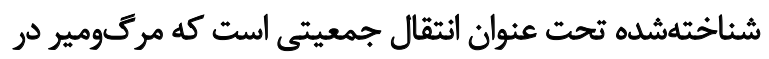

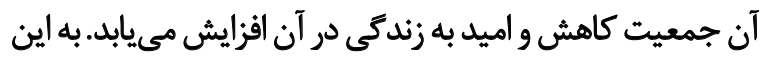

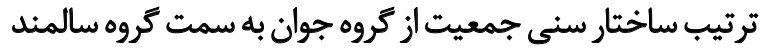

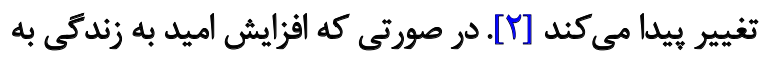

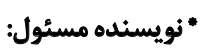

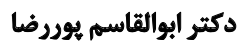

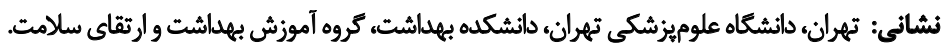

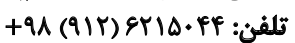

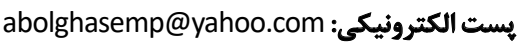


و عملكرد سلامتى را در سه نقطه بررسى كردند و ارتباطى مثبت

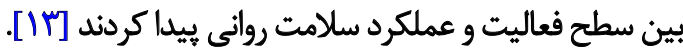
برخى از مطالعات مقطعى و طولى، روابط مثبت سركرمىهاى

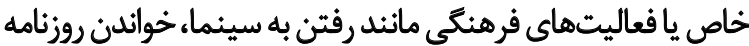

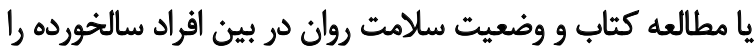

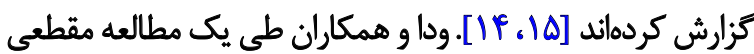

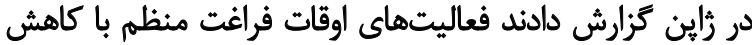

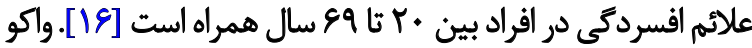

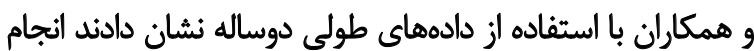

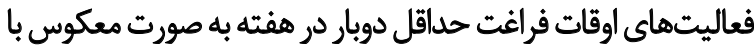

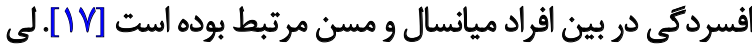

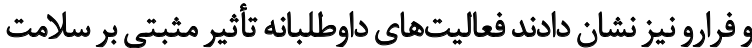

روانى افراد شصت سال و بالاتر دارند [1/1].

الكوى فراغت مناسب مى تواند تأثير مثبتى بر كيفيت زنداتى

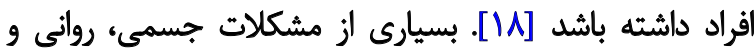

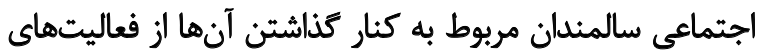

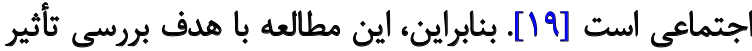

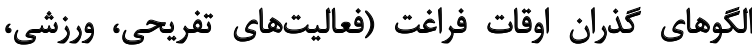

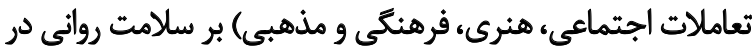

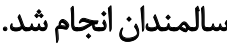

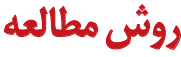

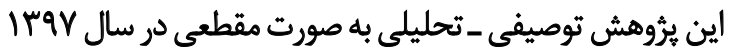

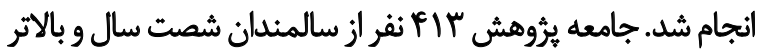

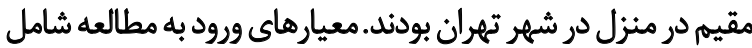

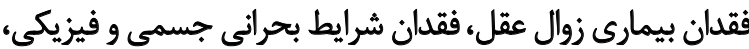

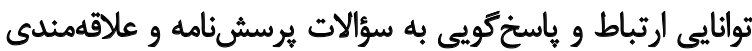

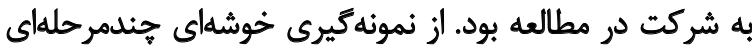

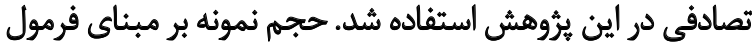

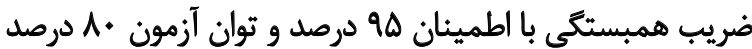

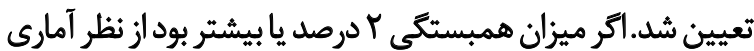

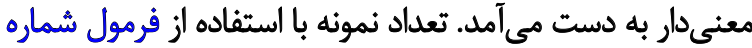
ا زير محاسبه شد:

$R=0.2$

$W=0.203$

$$
\begin{aligned}
& n=\frac{\left(z 1-\alpha /{ }_{2}+z_{1-\phi}\right) 2}{W 2}+3 \\
& n=\frac{(1.96+0.84) 2}{(0.203) 2}+3
\end{aligned}
$$

$n=192$
آن كشور سالمند خواهد بود [ه]] بر مبناى اطلاعات منتشرشده

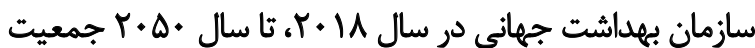

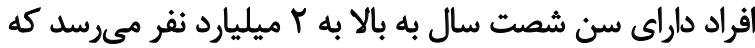

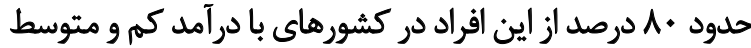

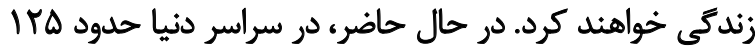

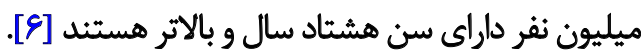
با توجه به رشد كاهنده جمعيت در كشور و روند رو به رشد بير

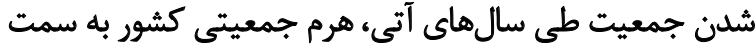

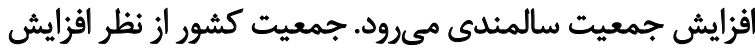

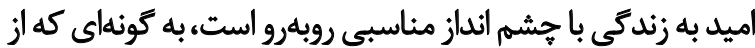

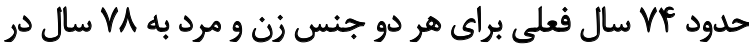

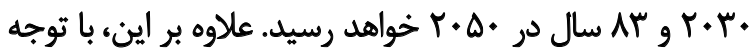

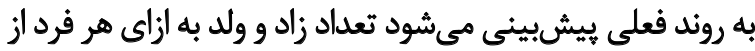

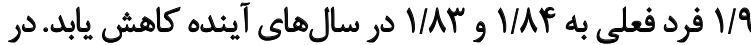

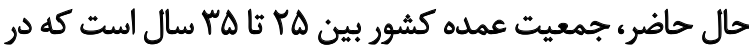

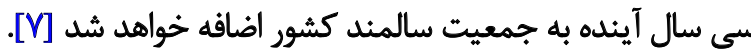

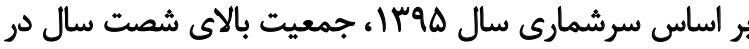

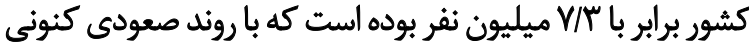

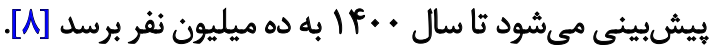
سالم بير شدن حق همه افراد بشر است و اين امر بر اهميت

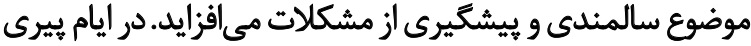

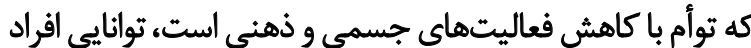

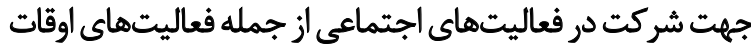

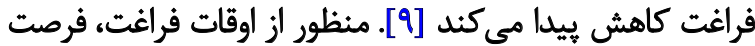

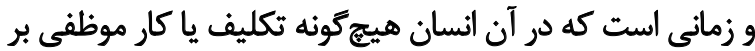

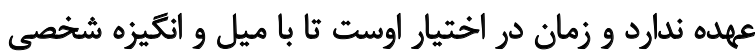

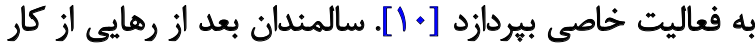

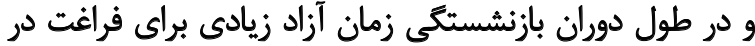

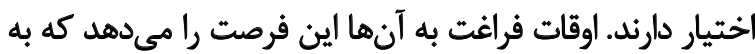

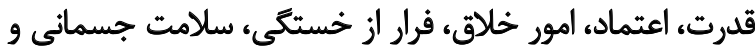

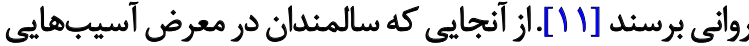

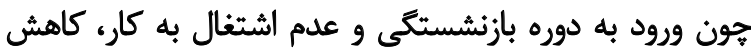

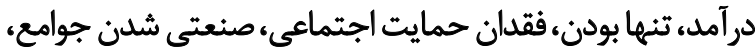

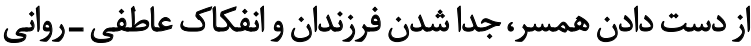

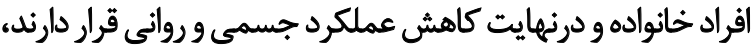

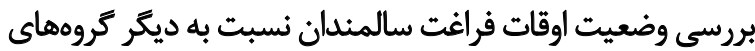

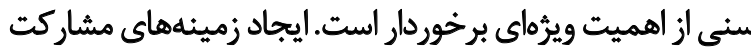

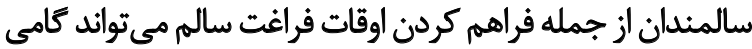
مؤثر در رفع اين خطرات و تهديدات باشد فردات فراءت مشاركت در فعاليتهاى فراغتى مي تواند سلامت روان در افراد

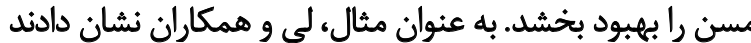

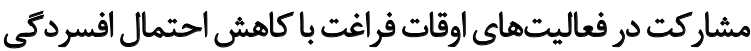

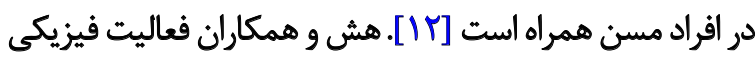




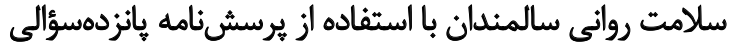

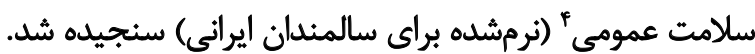

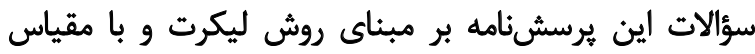

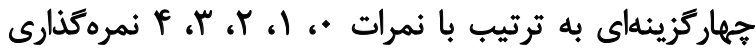

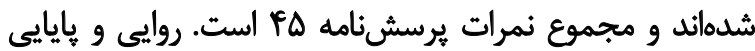

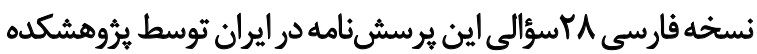

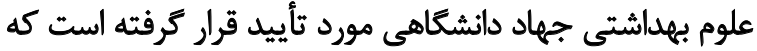

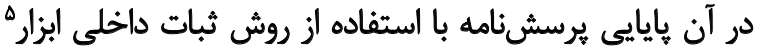

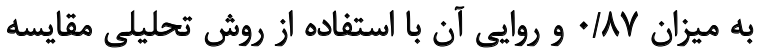

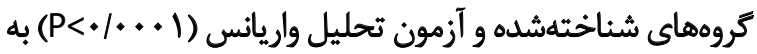

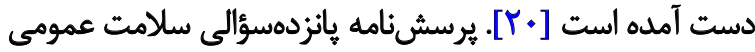

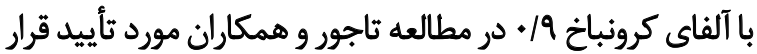

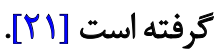

براى سنجش سلامت جسمى (به عنوان متغير مخدوش كنئده

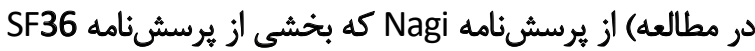

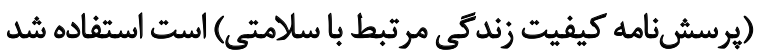

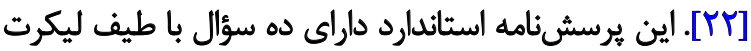

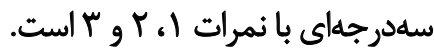

يرسش نامهها به صورت مصاحبه تكميل شدند. اتر در خانه

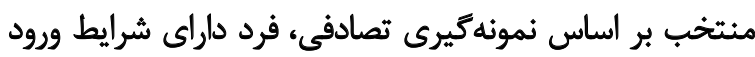

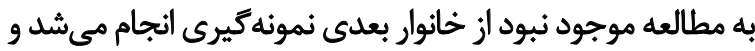

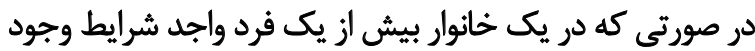

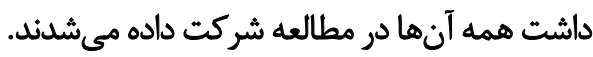

جهت تجزيه و تحليل دادهها از روشهاى آمار تونار توصيفى -

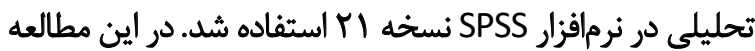

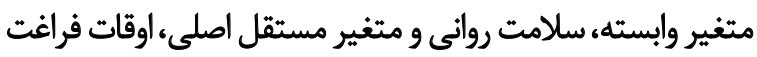
بود. در بخش توصيفى به توصيف فراواني و انحرافيته

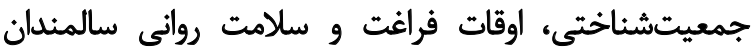

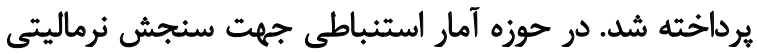

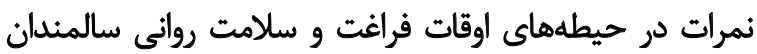

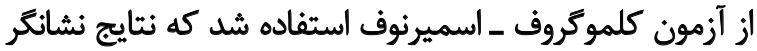

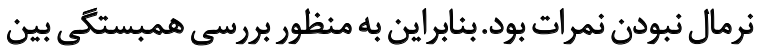

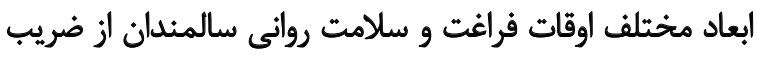

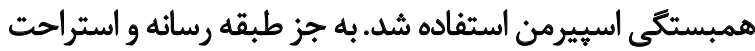

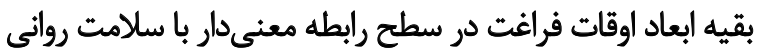

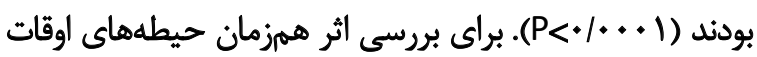

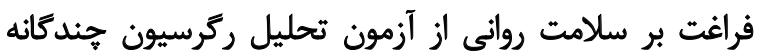

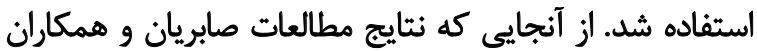

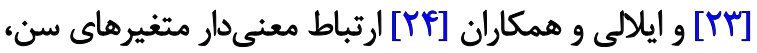

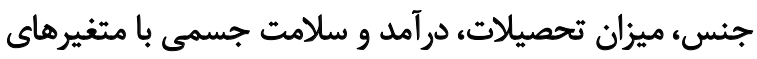
كذران اوقات فراغت و سلامت روان رانشان دران دادهاند، در اين مطالعه بانه

4. General Health Questionnaire (GHQ)

5. Internal Consistency
با توجه به اينكه روش نمونه ئيرى خوشهاى طبقهبندى فريده

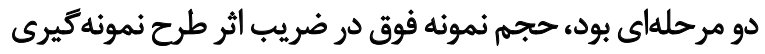

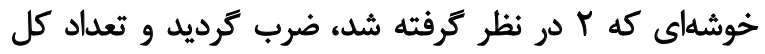

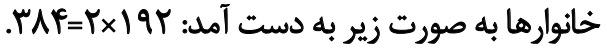

شركت كنندكان مورد نظر از شمال، جنوب، شرق، غرب و مركز

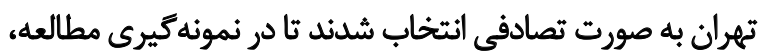

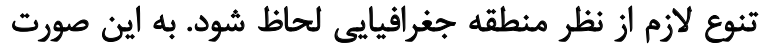

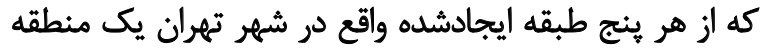

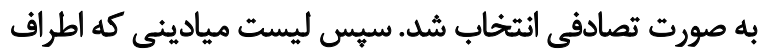

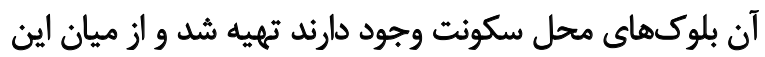

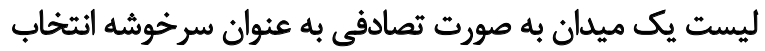

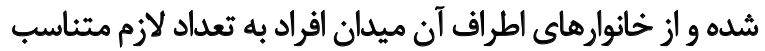

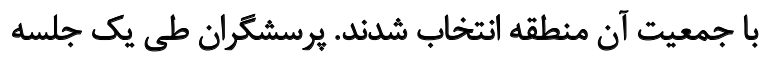

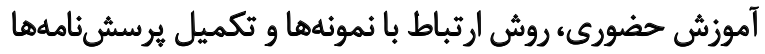

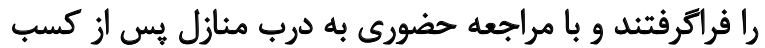

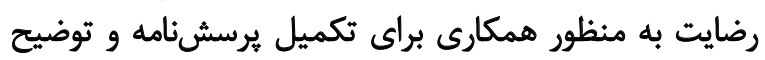

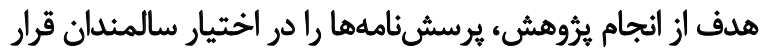

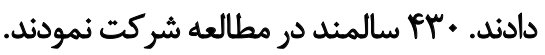

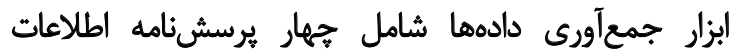

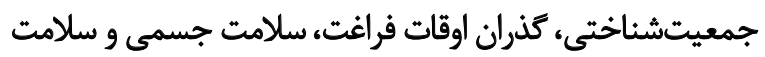

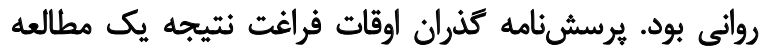

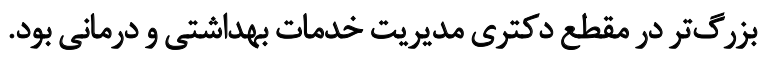

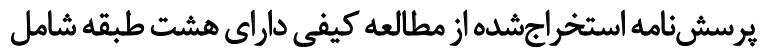

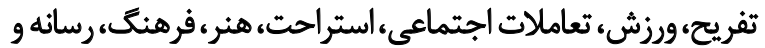

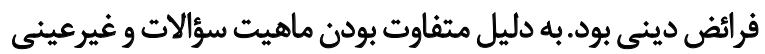

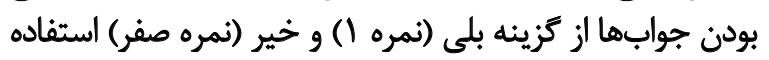

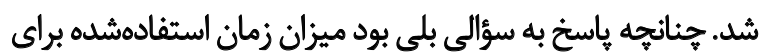

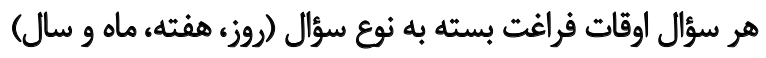

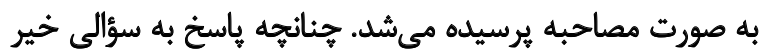

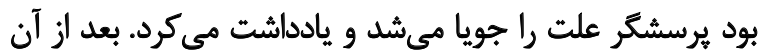

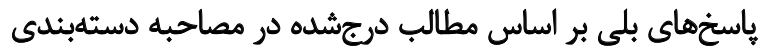

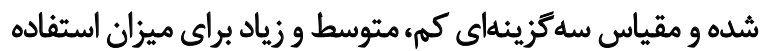

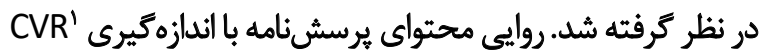

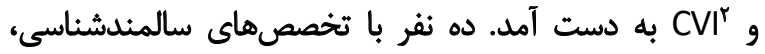

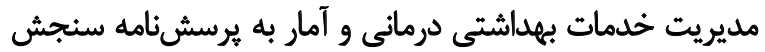

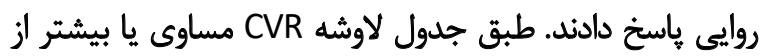

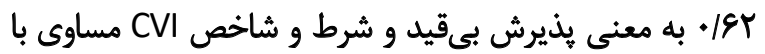

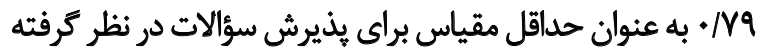

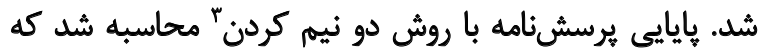

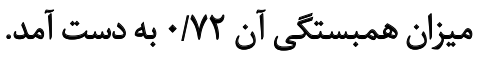


جدول ا. توصيف ويثركىهاي سالمثدان شركت كنئه در مطالعه به تفكيك جنسيت

\begin{tabular}{|c|c|c|c|c|}
\hline \multicolumn{3}{|c|}{ تعداد (درصد) } & \multirow{2}{*}{\multicolumn{2}{|c|}{ ويزؤكى جمعيثي }} \\
\hline كل & 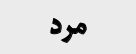 & زن & & \\
\hline $1.8(Y \Delta / F)$ & $P(M / E)$ & $\Delta A(1+/ *)$ & . & \\
\hline$r+\&(e q / V)$ & I. $(M E / g)$ & $1 . P(T \Delta / 1)$ & سو V. سال V & كروه سنى \\
\hline $1.1\left(M^{\prime} / K^{\prime}\right)$ & $0 \cdot(18 / 1)$ & D) $(1 / T / N)$ & 1) & \\
\hline$r q(N / 1)$ & $\operatorname{Ar}(F F / T)$ & $110(T V / A)$ & مثأهل & \\
\hline$r \varepsilon(\varepsilon / T)$ & $V(V / 8)$ & $19(4 / 9)$ & مجرد، جلانشهه & وضعيت تأهل \\
\hline $19(Y / D)$ & $1+(T / P)$ & $89(19 / 1)$ & بيوه & \\
\hline $8 \cdot(1 f / 4)$ & $\pi(r / 1)$ & $\operatorname{Pr}(11 / r)$ & ل بي سواد & \\
\hline $\operatorname{lre}(r \cdot / r)$ & $\Delta q(1 F / T)$ & $\operatorname{sv}(18 / \pi)$ & خواندن و نوشتن & \\
\hline$n^{n}(\mid V / \Delta)$ & $\pi(V / 9)$ & $f \cdot(q / q)$ & راهنمايى & ميزان تحصيلات \\
\hline$u(\pi / v)$ & $\Delta V(I r / A)$ & fi $(9 / 9)$ & دييلم & \\
\hline$\Delta \rho(\mid r / \Delta)$ & rA $(q / Y)$ & $M(F / T)$ & ليسانس و بالاتر & \\
\hline $\operatorname{Qr}(T Y / Y)$ & $\operatorname{le}(11 / 1)$ & $\operatorname{re}(11 / 1)$ & خوب & \\
\hline $\operatorname{rAT}$ (ENA) & $\ln (\pi / T)$ & $10 \cdot(r E / M)$ & هتوسط & وضعيت مالى \\
\hline 队ג (\&/) & $M(\Delta / \cdot)$ & $\operatorname{IV}(f / N)$ & ضعيف & \\
\hline
\end{tabular}

\section{ـ الم}

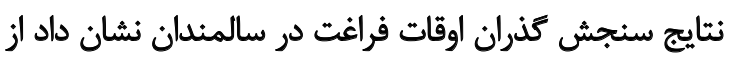

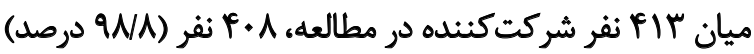

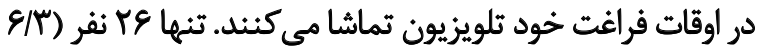

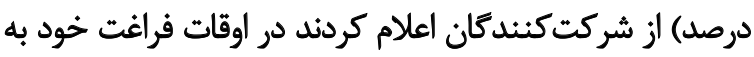

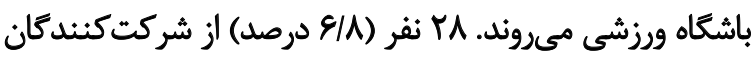

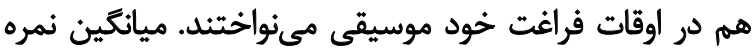

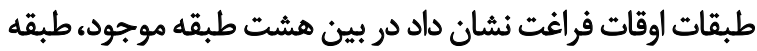

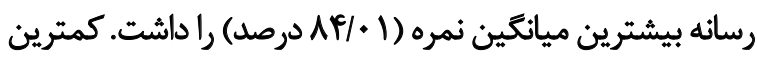

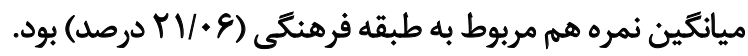

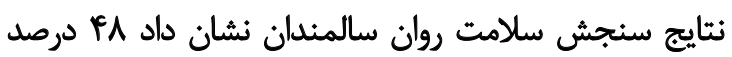

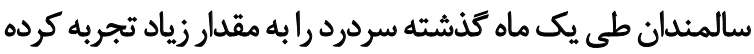

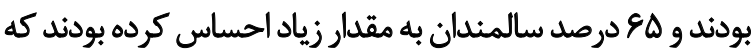
نقش مفيدى در زندگى ندارند.

Fتايج سنجش سلامت جسمى سالمندان نشان داد

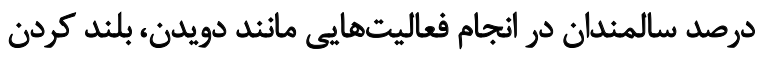

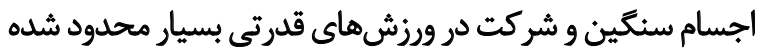

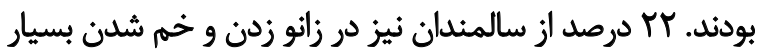
محدود شُده يودند.

نتايج آزمون تفاوت ميانگين نمرههاى حيطههاى اوقات فراغت
تأثير متغيرهاى مداخلهكر مانند سن، جنس، ميزان تحصيلات، وضعيت مالى و سلامت جسمى روى آناليزها كنترل شدئ مئد

ياقتهها

در تحقيق حاضر از مجموع · · Fr سالمند واردشده به مطالعه،

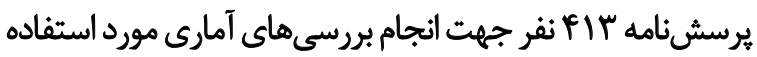

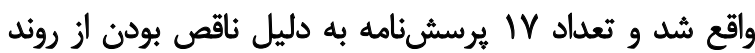

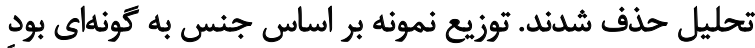

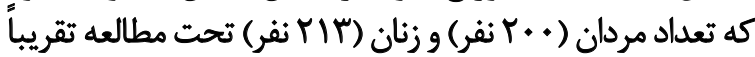

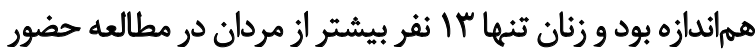

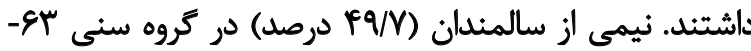

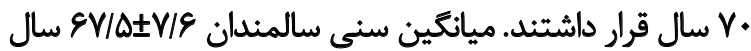

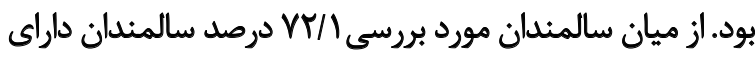

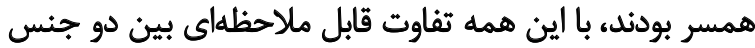

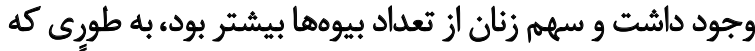

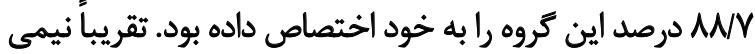

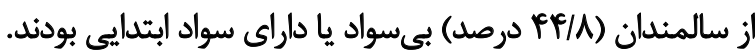

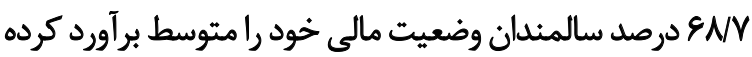

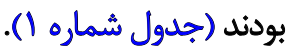


جدول r. نتايج آزمون همبستكى اسييرمن طبقات اوقات فراغت با سلامت روان (QHG)

\begin{tabular}{|c|c|c|}
\hline سطح معنى دارى & ضريب همبستكى & طبقات اوقات فراغت \\
\hline 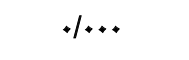 &.$- / T \Delta A$ & تفريح \\
\hline$\%$ & $-\cdot / r r s$ & ورزش \\
\hline .10 .1 &.$- / 194$ & تعاملات اجتماعي \\
\hline.$(\Delta)$ &.$/ \cdot \pi$ & استراحت \\
\hline .10. & $-\cdot / r \cdot V$ & 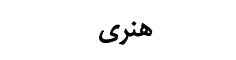 \\
\hline 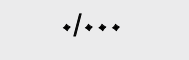 &.$- / 184$ & فرهنكي \\
\hline . MTr & -.1 .8 & رسانه \\
\hline .10 .5 &.$- / 1 f$. & فراثض مذهنى \\
\hline
\end{tabular}

L

اوقات فراغت و سلامت سالمندان (به علت نرمال نبودن نمرات) از

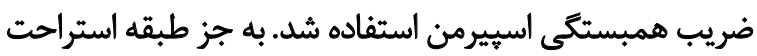

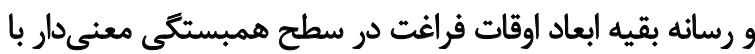
ابعاد سلامتى بودند (جدول شماره ؟).

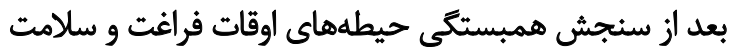

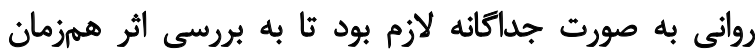

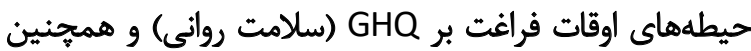

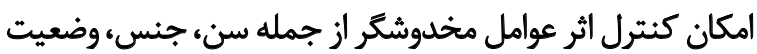

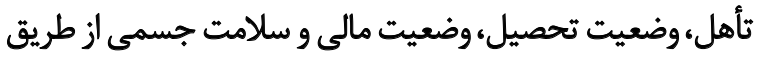

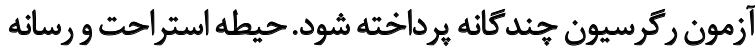

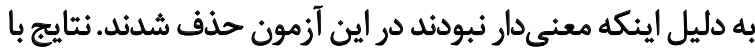

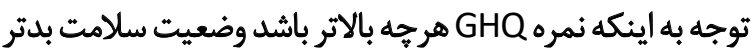
است به دست آمد (جدول شماره بَ).

مدل ا نتايج ركرسيون جند آنانه مربوط به طبقههاى اوقات

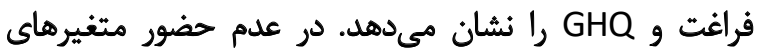

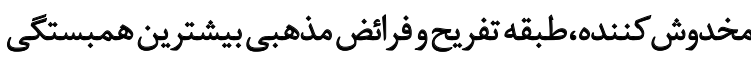
معكوس را با GHQ داشتند كه با توجه به ميزان Beta اثركنداري

$$
\text { فرائض مذهبى بيشتر بود. }
$$

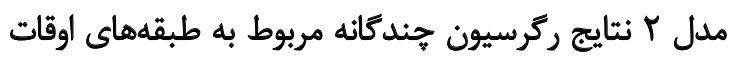

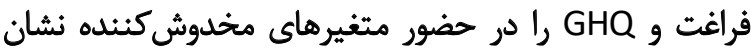

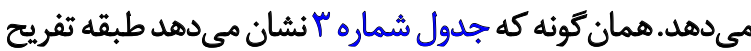

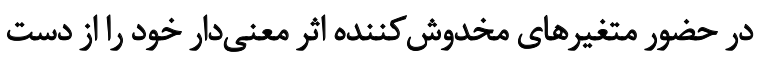

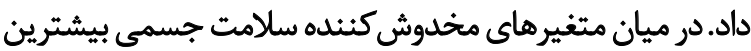

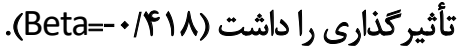

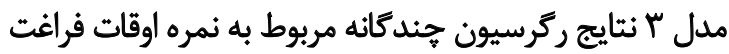

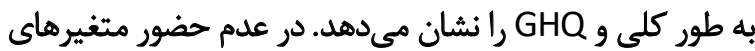

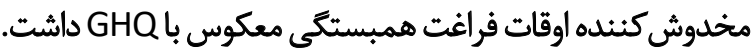

در دو جنس زن و مرد نشان داد مشاركت زنان در حيطههاي

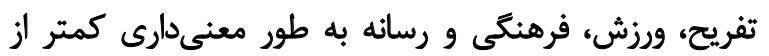

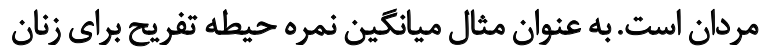

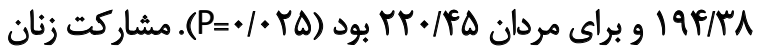

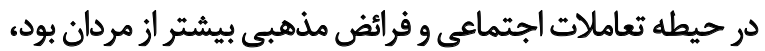

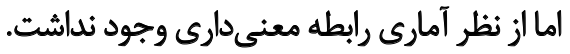

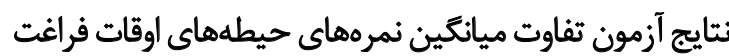

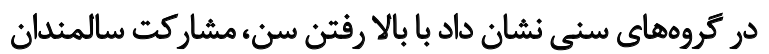

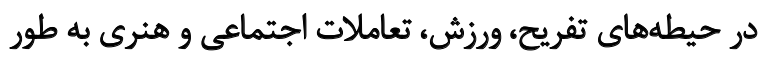

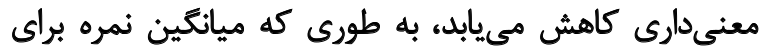

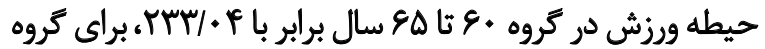

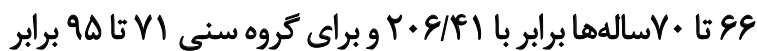

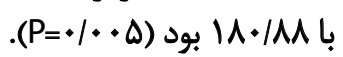

در رابطه با متغير وضعيت ثأهل مطالعه ما نشان داد مشاركت

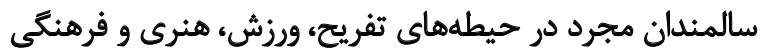

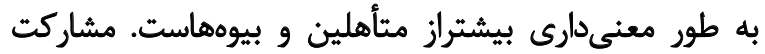

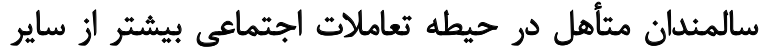

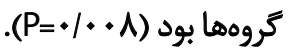

در اين مطالعه جهت سنجش سلامت بر مبناى سن و جنس

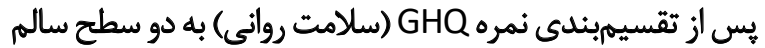

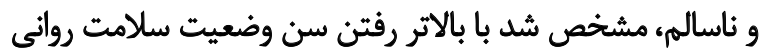

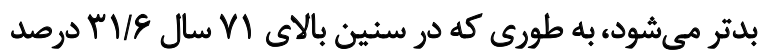

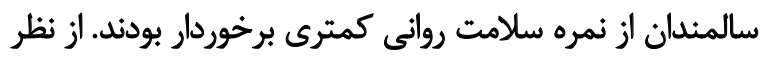

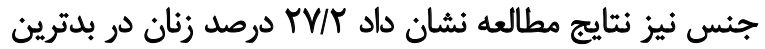

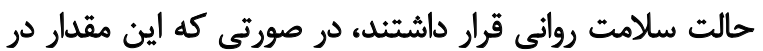

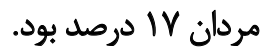
در يُروهش حاضر جهت بررسى همبستكى بين ابعاد مختلف 
نشان داد سالمندان ايرانى در اوقات فراغت خود به انجام فرائض

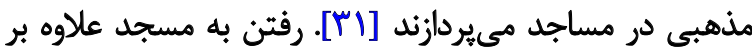

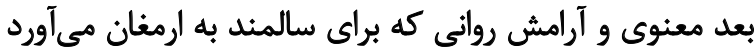

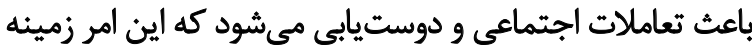
مشاركت سالمندان در فعاليتهاي فراغتى را فراهم ميت كي كند.

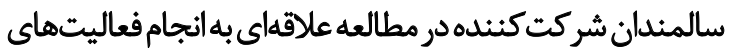
هنرى در اوقات فراغت خود نداشتئد كه نتيجه مطالعه جنئك

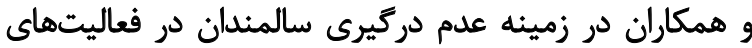

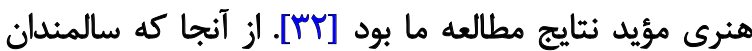

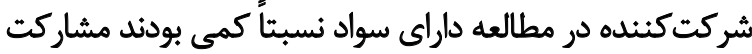

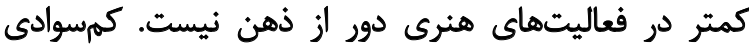

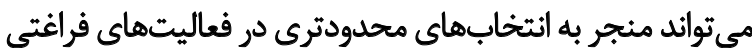

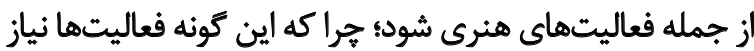

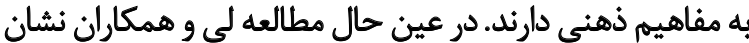

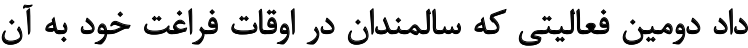

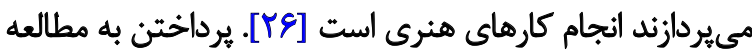

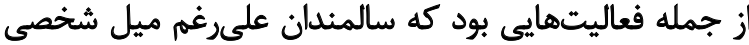

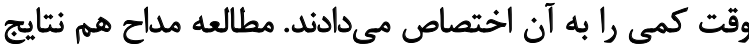

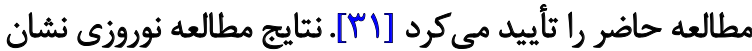

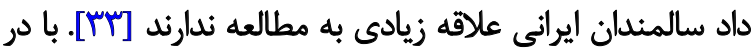

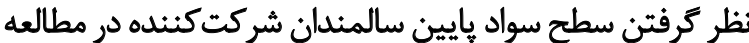

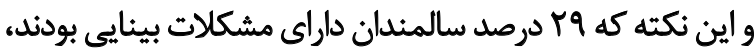

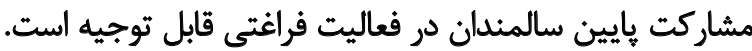

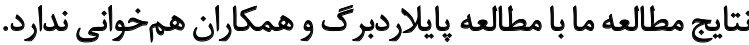

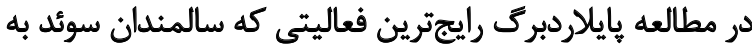

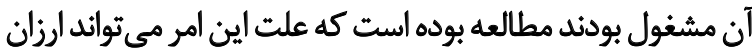

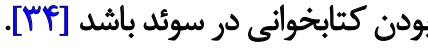

روند مطالعه ما به گَونهاي بود كه سالمندانى كه به سؤالات جواب

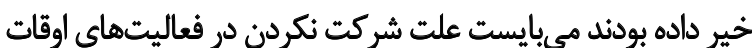

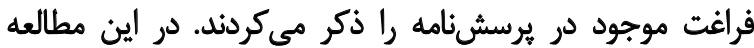

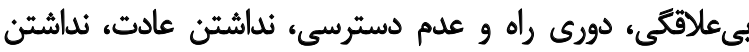

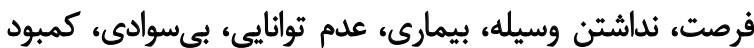

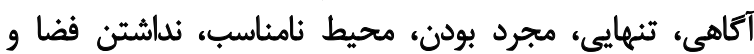

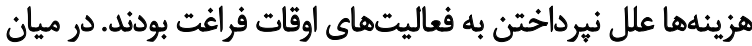

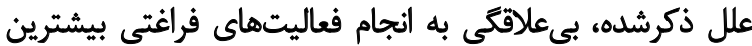

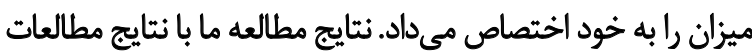

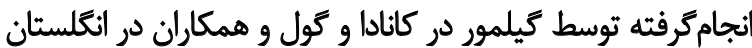

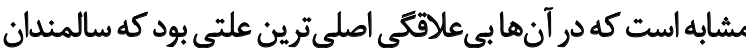

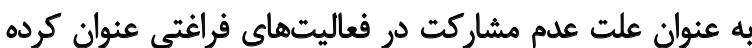

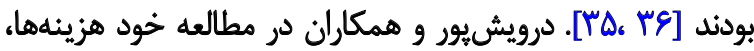

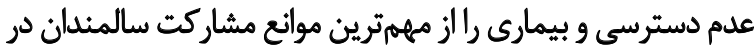

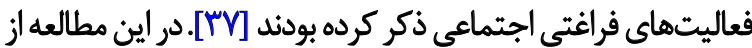

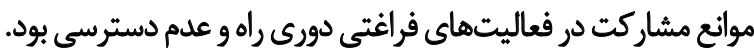

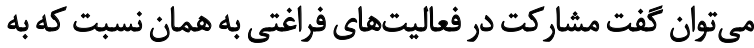

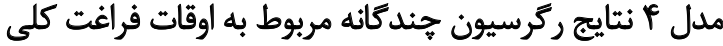

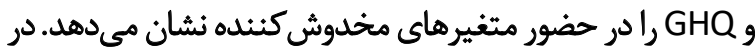

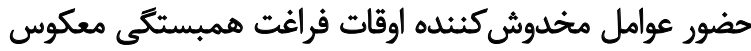

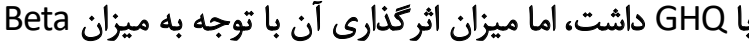

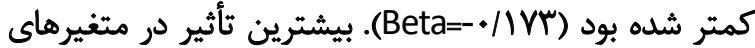

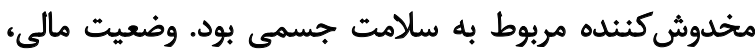
وضعيت تحصيلى و سن هم از عوامل تأثيركذار بودند.

بحث

اين بثروهش با هدف تعيين ارتباط بين روش هاى كذران اوقات

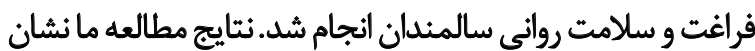

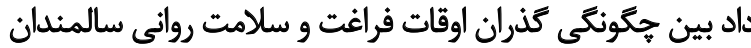

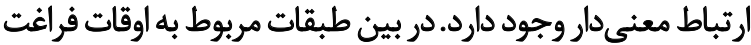

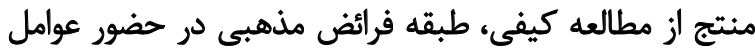

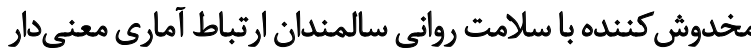

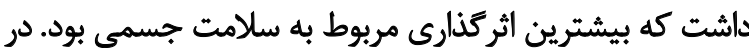

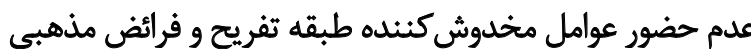

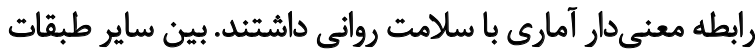
وقات فراغت و سلامت روان رابطه مهمى ديله نشا نشد.

يافتههاي مطالعه نشان داد فعاليت فراغتى رايج در سالمندان

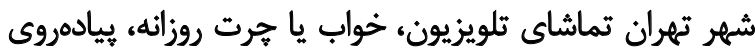

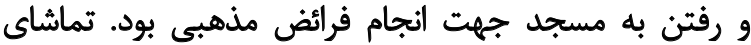

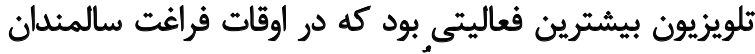

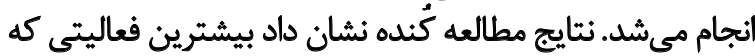

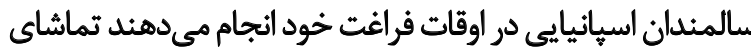

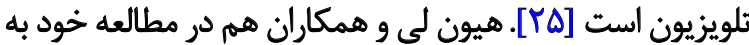

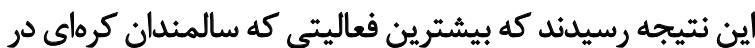

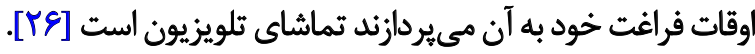

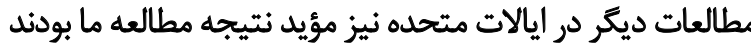

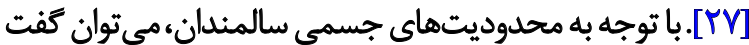

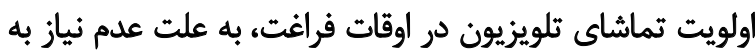

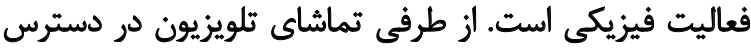

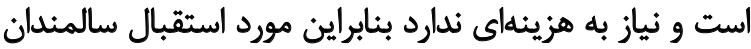

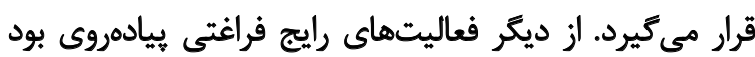

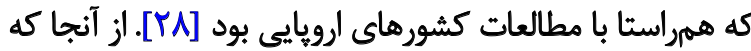

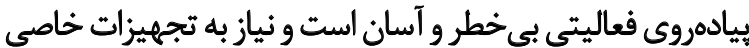

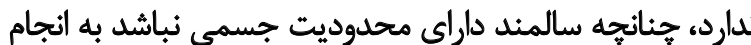

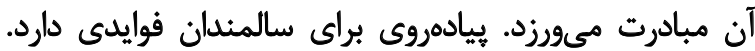

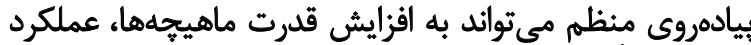

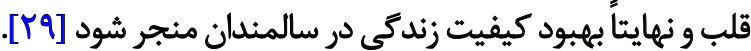

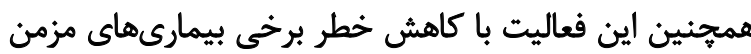

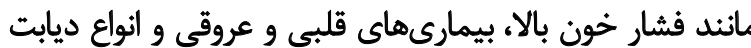

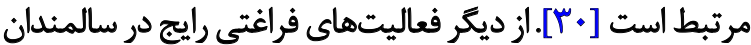

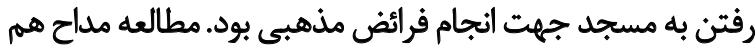


جHQ جدول ب. نتايج ركرسيون جندكانه مربوط به طبقات اوقات فراغت

\begin{tabular}{|c|c|c|c|c|c|c|c|c|c|c|c|c|}
\hline \multicolumn{3}{|c|}{ مدل ع } & \multicolumn{3}{|c|}{ مدل } & \multicolumn{3}{|c|}{ مدل r } & \multicolumn{3}{|c|}{ مدل 1} & \multirow{2}{*}{ هتغيرها } \\
\hline $\mathbf{F}$ & $\mathbf{P}$ & Beta & $\mathbf{F}$ & $\mathbf{P}$ & Beta & $\mathbf{F}$ & $\mathbf{P}$ & Beta & $\mathbf{F}$ & $\mathbf{P}$ & Beta & \\
\hline & - & - & & - & - & & . NE . & -.1 .19 & & I.rE & -+||$\Delta \mid$ & تفريح \\
\hline & - & - & & - & - & &.$/ 9 W$ & $.1 .0 Y$ & & . &.$- / . V \Delta$ & ورزش \\
\hline & - & - & & - & - & &.$/ A M$ &.$- / .4 A$ & & .1898 &.$- / \cdot M$ & تعاملات اجتماعي \\
\hline & - & - & & - & & $I r / I V$ & $.1 . \mathrm{V}$ & -.1 .94 & $\Delta H T / N$ & &.$- / .97$ & هنرى \\
\hline & - & - & & - & - & & . lare & $-* / * T$ & & זו"יו. & $-* / * \Delta r$ & فرهنكى \\
\hline & - & - & & - & - & & $1+r$. &.$- / .97$ & & $.1+10$ &.$- / M V$ & فرائض مذهبي \\
\hline \multirow[t]{7}{*}{$r E / T E$} & $\%$ &.$- / 1 V^{2}$ & $\Delta V / M$ &. &.$-|Y \Delta|$ & & - & - & & - & - & نمره كلى اوقات \\
\hline &.$/ T A A$ & $-+1+\Delta \Delta$ & & - & - & & עזM/. & $-4+8$ & & - & - & جنس \\
\hline & $+1 \Delta 59$ & $-+1+r V$ & & - & - & &.$/ 49$ & $-t /+m e$ & & - & - & سن \\
\hline & .1 .81 & $\cdot 1 \cdot 19$ & & - & - & & /. & .1 .99 & & - & - & وضعيت ثأهل \\
\hline &.$/ M^{2} q$ & $.1 \cdot 18$ & & - & - & &.$/ M W$ & $.1 \cdot 10$ & & - & - & وضعيت تحصيلى \\
\hline & $.1 . r V$ & .1 .94 & & - & - & & .1 .18 & $\cdot 11 \cdot 1$ & & - & - & وضعيت هالى \\
\hline & $+1+\ldots$ & $-+/ f i f$ & & - & - & & $\%$ & $-4 / 411$ & & - & - & سلامت جسمى \\
\hline
\end{tabular}

L

برخى از اين فضاهاى تفريحى و ورزشى اساساً مردانه هستند.

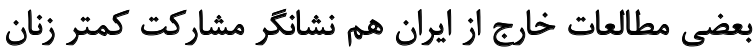

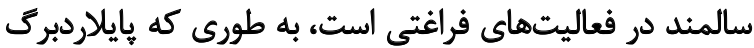

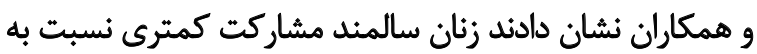

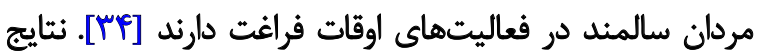

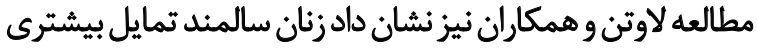

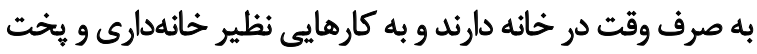

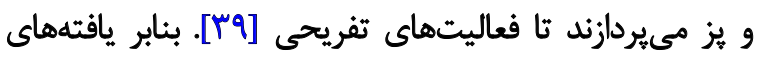

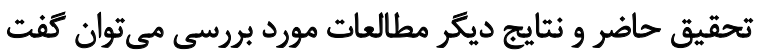

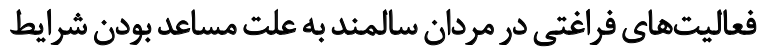

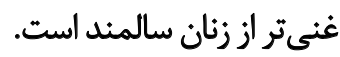

نتايج اين يُروهش نشان داد اوقات فراغت با سلامت روان

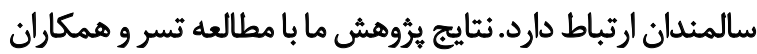

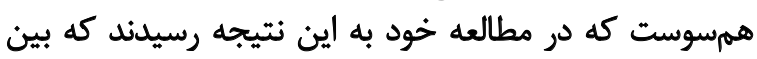

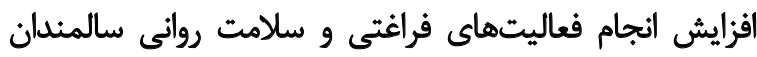

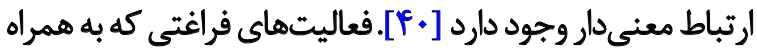

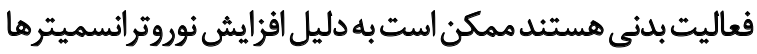

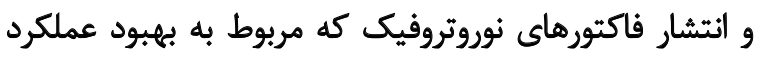

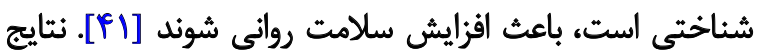

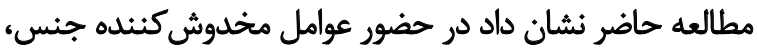

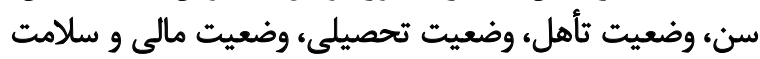

قصد و علاقه فرد بستگى دارد به شرايط محيط و امكانات نيز بستخكى

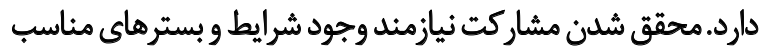

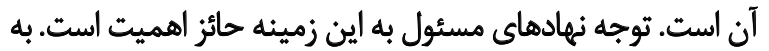

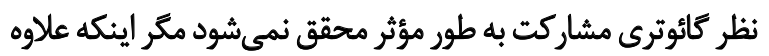

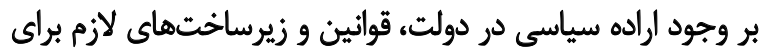
ترويج مشاركت موجود باشد.

نتايج مطالعه نشان داد مشاركت زنان سالمند در حيطه ورهاى

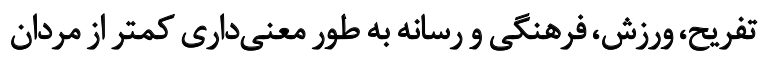

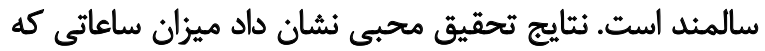

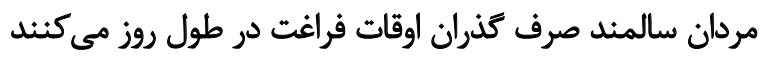

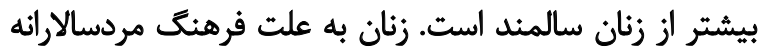

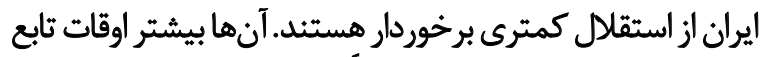

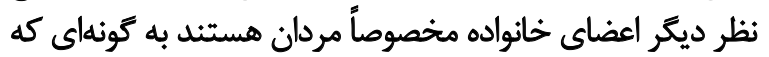

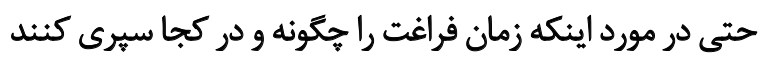

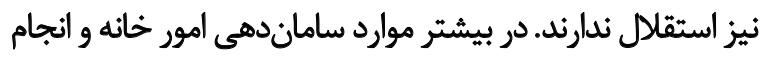

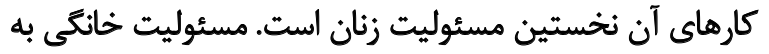

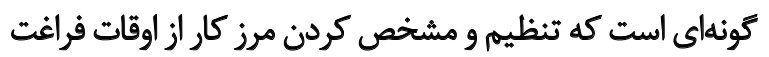

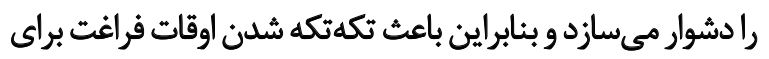

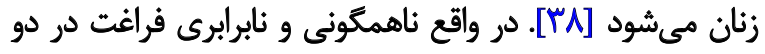

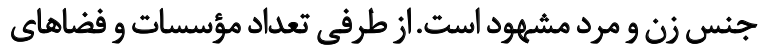

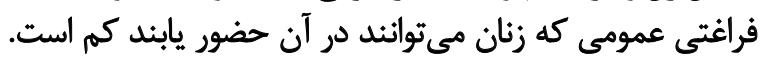


درمجموع،نتايج تحقيق حاضر همسو با ييشيئه نظرى و تجربى رئ

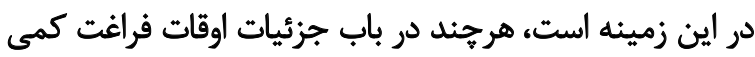

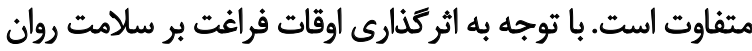

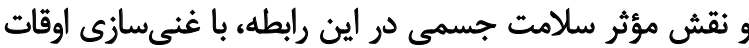

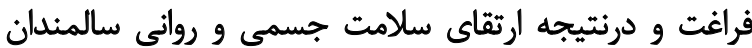

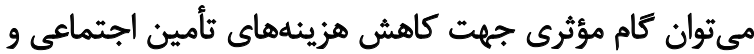

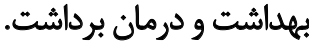

مهلدوديت

از جمله محدوديتهاي مهمم تحقيق حاضر، سنجش مفاهيم

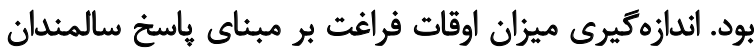

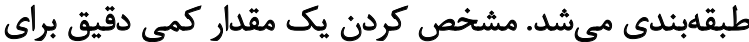

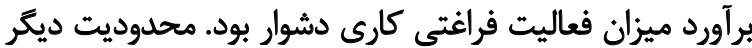

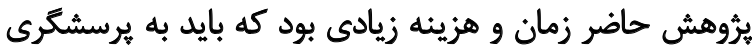

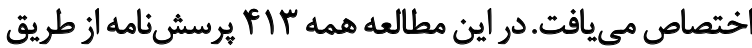

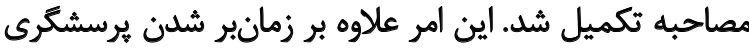

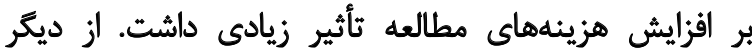

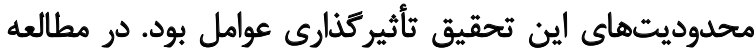

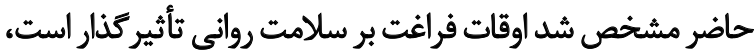
اما مي توان ارتباط عكس هم در اين زمينه برقرار كرد.

يويُشنهادات بروهيشي

يرؤشش حاضر بر مبناى روش كمى و به صورت بيمايش در

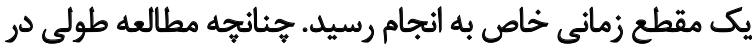

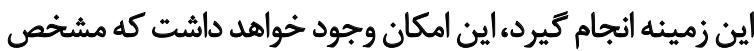

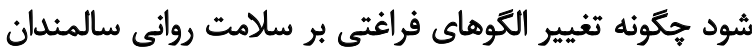

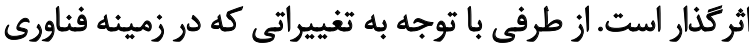

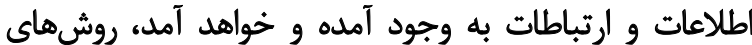

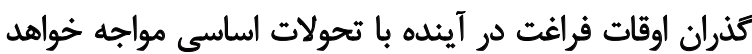

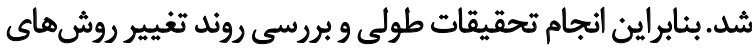

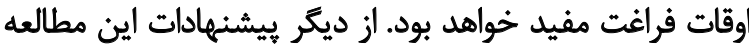

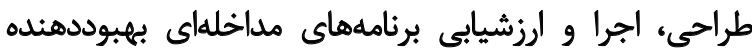

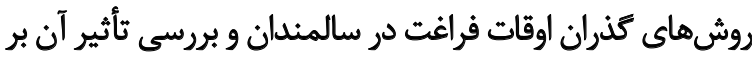
سلامت روانى سالمندان است.

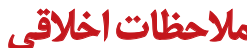

\section{يبيروى أز اصول الخلاق يثوهش}

اين مقاله مصوب دانشكده بهداشت دانشعاه علوميزشكى تهران با كد اخلاق IR.TUMS.VCR.REC.1395.320 است بعاله

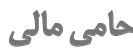

ايـن يُروهـش هيجگَونه كمـك مالـى از سـازمانيهاى دولتى،

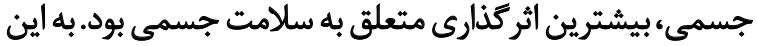

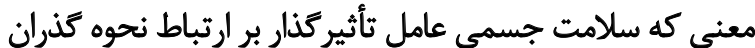

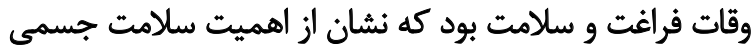

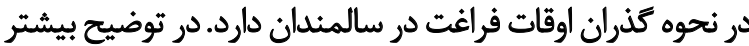

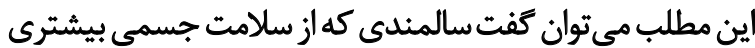

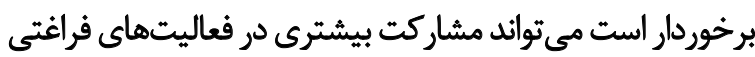

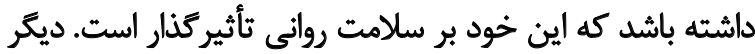

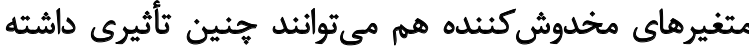

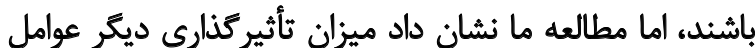

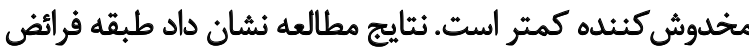

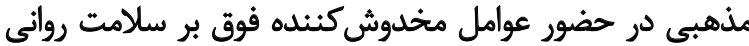

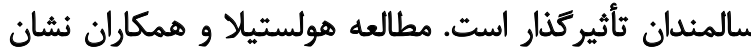

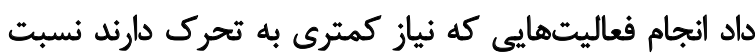

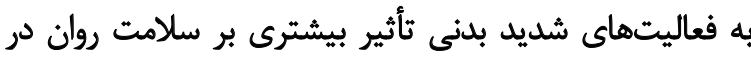

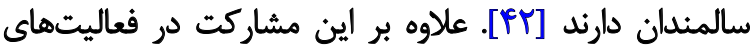

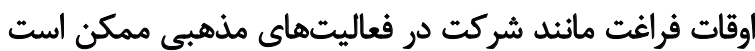

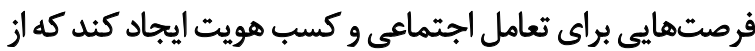

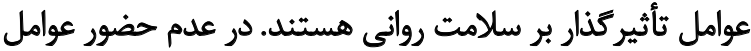

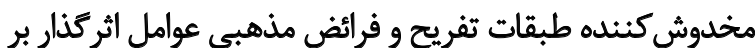

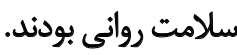

\section{نتيجلكيرى نهايي}

نتايج مطالعه نُشان داد سلامت جسمى عامل اثر كذار در رابطه

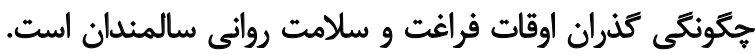

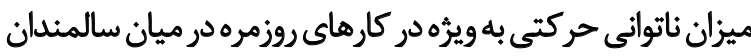

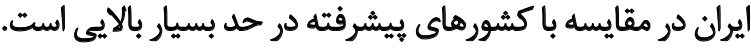

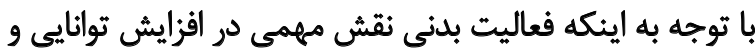

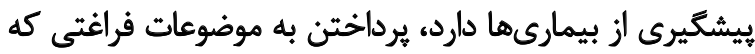

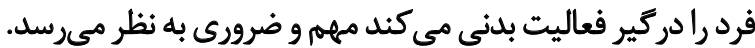

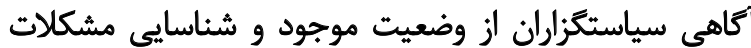

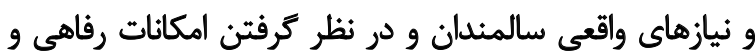

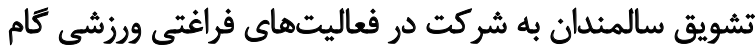

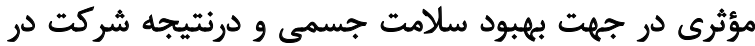

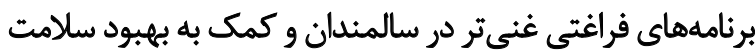

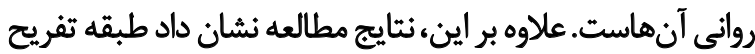

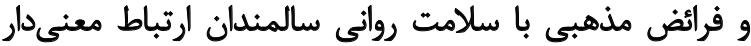

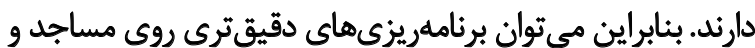

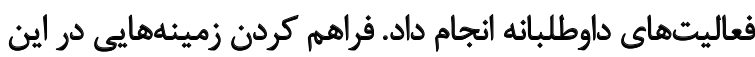

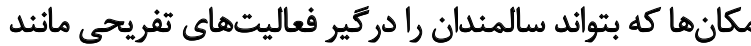

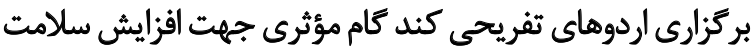

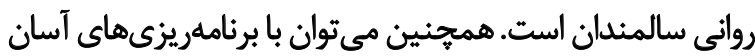

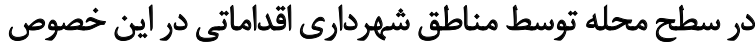

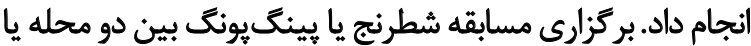

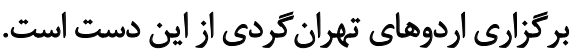




$$
\begin{aligned}
& \text { خصوصى و غيرانتفاعى دريافت نكرده اسـت. } \\
& \text { مشاركت نويسندكًان } \\
& \text { تمـام نويســندكان در آمادهســازى ايــن مقالـه مشــاركت } \\
& \text { داشــتهاند. } \\
& \text { تعارض مناقع }
\end{aligned}
$$

بنابر اظهار نويسندكان، اين مقاله تعارض منافع ندارد. 


\section{References}

[1] Jandaghi J, Majd M, Maghsodnya S, Froghan M, Rhmani K, Fariver F, et al. Revision of elderly medicine. Semnan: Semnan University of Medical Sciences. 2003.

[2] Kaveh Firouz Z. [The study of the effect of socio-demographic structures on the elderly 's quality of life in Tehran (Persian)] [PhD. dissertation]. Tehran: University of Tehran; 2011.

[3] Joghataei MT, Nejati V. [Assessment of health status of elderly people in the city of Kashan (Persian)]. Salmand: Iranian Journal of Ageing. 2006; 1(1):3-10. http:/ / salmandj.uswr. ac.ir/article-1-26-en.htm

[4] Momeni Kh, Karimi H. [Comparison of mental health between elderly admitted in sanitarium with elderly in sited in personal home (Persian)]. Journal of Kermanshah University of Medical Sciences. 2011; 14(4):e79446. https://sites. kowsarpub.com/jkums/articles/79446.html

[5] Dadkhah A. [Review of aged rendered services in USA and Japan and guidelines for Iran aging strategic plan (Persian)]. Salmand: Iranian Journal of Ageing. 2007; 2(1):166-76. http:/ / salmandj.uswr.ac.ir/browse.php?a_id=1236\&sid=1\&slc_ lang $=\mathrm{fa}$

[6] World Health Organization. Aging and health [Internet]. 2018 [Updated 2018 February 5]. Available from: https:// www.who.int/news-room/fact-sheets/detail/ageing-andhealth

[7] Rezaii E. [The effect of demographic changes on government social expenditure and life cycle expenditure in Iran economy (Persian)]. Quarterly Journal of Fiscal and Economic Policies. 2016; 3(11):62-84. http:/ / qjfep.ir/article-1-341-en. html

[8] Yousefi H, Abedi HA, Yarmohammadian MH, Elliott D. Comfort as a basic need in hospitalized patients in Iran: A hermeneutic phenomenology study. Journal of Advanced Nursing. 2009; 65(9):1891-8. [DOI:10.1111/j.13652648.2009.05026.x] [PMID]

[9] Ghazi Moradi H. [Work and leisure in Iranian people (Persian)]. $3^{\text {rd }}$ ed. Tehran: Ameh Book; 2012. http://opac.nlai.ir/ opac-prod/bibliographic/2069378

[10] Abedi HA, Lali M, Keyvanniya Sh, Nikbakht A. [Life experiences of older people who are used to spend their time in parks (Persian)]. Journal of Qualitative Research in Health Sciences. 2013; 2(2):184-93. http://eprints.kmu.ac.ir/30687/

[11] Lampinen P, Heikkinen RL, Kauppinen M, Heikkinen E. Activity as a predictor of mental well-being among older adults. Aging and Mental Health. 2006; 10(5):454-66. [DOI:10.1080/13607860600640962] [PMID]

[12] Lee HY, Yu CP, Wu CD, Pan WC. The effect of leisure activity diversity and exercise time on the prevention of depression in the middle-aged and elderly residents of Taiwan. International Journal of Environmental Research and Public Health. 2018; 15(4):654. [DOI:10.3390/ijerph15040654] [PMID] [PMCID]

[13] Heesch KC, van Uffelen JG, van Gellecum YR, Brown WJ. Dose-response relationships between physical activity, walking and health-related quality of life in mid-age and older women. Journal of Epidemiology and Community Health. 2012; 66(8):670-7. [DOI:10.1136/jech-2011-200850] [PMID]
[14] Wolin KY, Glynn RJ, Colditz GA, Lee IM, Kawachi I. Long term physical activity patterns and health-related quality of life in U.S. women. American Journal of Preventive Medicine. 2007; 32(6):490-9. [DOI:10.1016/j.amepre.2007.02.014] [PMID] [PMCID]

[15] Barcelos-Ferreira R, Nakano EY, Steffens DC, Bottino CM. Quality of life and physical activity associated to lower prevalence of depression in community-dwelling elderly subjects from Sao Paulo. Journal of Affective Disorders. 2013; 150(2):616-22. [DOI:10.1016/j.jad.2013.02.024] [PMID]

[16] Wada K, Satoh T, Tanaka K, Tsunoda M, Aizawa Y. Associations of depressive symptoms with regular leisure activity and family social support among Japanese workers. Industrial Health. 2007; 45(1):181-5. [DOI:10.2486/indhealth.45.181] [PMID]

[17] Wakui T, Saito T, Agree EM, Kai I. Effects of home, outside leisure, social, and peer activity on psychological health among Japanese family caregivers. Aging \& Mental Health 2012; 16(4):500-6. [DOI:10.1080/13607863.2011.644263] [PMID] [PMCID]

[18] Li Y, Ferraro KF. Volunteering and depression in later life Social benefit or selection processes? Journal of Health and Social Behavior. 2005; 46(1):68-84. [DOI:10.1177/00221465050460 0106] [PMID]

[19] Rahimi A, Anoosheh M, Ahmadi F, Foroughan M. [Tehranian elderly people's experiences about leisure time activities and recreations (Persian)]. Salmand: Iranian Journal of Ageing 2010; 5(1):61-77. http://salmandj.uswr.ac.ir/article-1-295-en. html

[20] Seyfzadeh A, Hagighatian M, Mohajerani AA. [The relationship between social isolation and health among the Tehranian elderly (Persian)]. Journal of Education and Community Health. 2017; 4(3):19-25. [DOI:10.21859/jech.4.3.19]

[21] Tajvar M, Grundy E, Fletcher A. Social support and mental health status of older people: A population-based study in Iran-Tehran. Aging \& Mental Health. 2018; 22(3):344-53. [DOI 10.1080/13607863.2016.1261800] [PMID]

[22] Montazeri A, Goshtasebi A, Vahdaninia M, Gandek B. The Short Form Health Survey (SF-36): Translation and validation study of the Iranian version. Quality of Life Research. 2005; 14(3):875-82. [DOI:10.1007/s11136-004-1014-5] [PMID]

[23] Saberian M, Haji Aghajani S, Ghorbani R. [Study of the mental status of the elderly and its relationship with leisure time activities (Persian)]. Journal of Sabzevar University of Medical Sciences. 2004; 10(4):53-60. https://www.sid.ir/fa/journal/ ViewPaper.aspx?ID=32870

[24] Ilali ES, Bashkani M, Jahanbakhsh F. [A review of the effect of leisure on depression in the elderly (Persian)]. Clinical Excellence. 2018; 8(2):50-60. http://ce.mazums.ac.ir/article1-411-en.html

[25] Conde MD. Leisure patterns and needs of the elderly in rural Galicia (Spain). Educational Gerontology. 2012; 38(2):138-45. [DOI:10.1080/03601277.2010.515894]

[26] Lee JH, Lee JH, Park SH. Leisure activity participation as predictor of quality of life in Korean Urban-dwelling elderly. Occupational Therapy International. 2014; 21(3):124-32. [DOI:10.1002/oti.1371] [PMID] 
[27] Krantz-Kent R, Stewart J. How do older Americans spend their time? Monthly Labor Review. 2007; 130:8. https:// heinonline.org/hol-cgi-bin/get_pdf.cgi?handle=hein.journals $/$ month130\&section $=41$

[28] Triadó C, Villar F, Solé C, Celdrán M, Osuna MJ. Daily activity and life satisfaction in older people living in rural contexts. The Spanish Journal of Psychology. 2009; 12(1):23645. [DOI:10.1017/S1138741600001645] [PMID]

[29] Deslandes A, Moraes H, Ferreira C, Veiga H, Silveira $\mathrm{H}$, Mouta R, et al. Exercise and mental health: Many reasons to move. Neuropsychobiology. 2009; 59(4):191-8. [DOI:10.1159/000223730] [PMID]

[30] Tudor-Locke C, Bassett DR Jr. How many steps/day are enough? Preliminary pedometer indices for public health. Sports Medicine. 2004; 34(1):1-8. [DOI:10.2165/00007256200434010-00001] [PMID]

[31] Madah SB, Emami A, Rahgozar M, Foroughan M, Norouzi Tabrizi K, Mohammadi F, et al. [The status of social and leisure time activities in the elderly residing in Iran and Sweden (Persian)]. Salmand: Iranian Journal of Ageing. 2008; 3(2):597606. http:// salmandj.uswr.ac.ir/article-1-95-en.html

[32] Cheung MC, Ting W, Chan LY, Ho KS, Chan WM. Leisure participation and health-related quality of life of community dwelling elders in Hong Kong. Asian Journal of Gerontology \& Geriatrics. 2009; 4(1):15-23. http://www.hkag.org/Publications/AJGG/V4N1/p15_2009-46-OA.pdf

[33] Norouzi Tabrizi K. [Design, implementation and evaluation of a care model on the community of elderly people living in Tehran (Persian)] [PhD. dissertation]. Tehran: Tarbiat Modares University; 2006.

[34] Paillard-Borg S, Wang HX, Winblad B, Fratiglioni L. Pattern of participation in leisure activities among older people in relation to their health conditions and contextual factors: A survey in a Swedish urban area. Ageing \& Society. 2009; 29(5):803-21. [DOI:10.1017/S0144686X08008337]

[35] Gilmour H. Social participation and the health and wellbeing of Canadian seniors. Health Reports. 2012; 23(4):23-32. [PMID]

[36] Goll JC, Charlesworth G, Scior K, Stott J. Correction: Barriers to social participation among lonely older adults: The influence of social fears and identity. PLoS One. 2018; 13(7):e0201510. [DOI:10.1371/journal.pone.0201510] [PMID] [PMCID]

[37] Darvishpoor Kakhki A, Abed Saeedi Z, Abbaszadeh A. [Social participation, barriers, and related factors in older people in Tehran (Persian)]. Journal of Health Promotion Management. 2014; 3(4):65-73. http://jhpm.ir/article1-346-en.html

[38] Ebrahimi GA, Razeghi N, Moslemi Ptrod R. [Social factors affecting leisure A case study of the 15-64 year- old in the city of Jouybar, Mazandaran, Iran (Persian)]. Journal of Applied Sociology. 2012; 22(4):71-98. https://jas.ui.ac.ir/article_18244.html?lang=en

[39] Lawton MP, Brody EM. Assessment of older people: Self-maintaining and instrumental activities of daily living. The Gerontologist. 1969; 9(3 Pt 1):179-86. [DOI:10.1093/geront/9.3_Part_1.179] [PMID]
[40] Tessier S, Vuillemin A, Bertrais S, Boini S, Le Bihan E Oppert JM, et al. Association between leisure-time physical activity and health-related quality of life changes over time. Preventive Medicine. 2007; 44(3):202-8. [DOI:10.1016/j. ypmed.2006.11.012] [PMID]

[41] Melzer I, Benjuya N, Kaplanski J. Effects of regular walking on postural stability in the elderly. Gerontology. 2003; 49(4):240-5. [DOI:10.1159/000070404] [PMID]

[42] Holstila A, Mänty M, Rahkonen O, Lahelma E, Lahti J. Changes in leisure-time physical activity and physical and mental health functioning: A follow-up study. Scandinavian Journal of Medicine \& Science in Sports. 2017; 27(12):1785-92. [DOI:10.1111/sms.12758] [PMID] 